\title{
Étude sur modele physique et numerique des evacuateurs de crue et des fosses d'erosion du barrage de Koman en Albanie
}

\author{
Giovanni DE CESARE ${ }^{1}$, Milad DANESHVARI ${ }^{2}$, Matteo FEDERSPIEL ${ }^{3}$, \\ Mathias MALQUARTI ${ }^{4}$, Gaël EPELY CHAUVIN ${ }^{5}$, Anton SCHLEISS $^{6}$
}

\author{
1. Laboratoire de Constructions Hydrauliques (LCH), Ecole Polytechnique Fédérale de Lausanne (EPFL), Station 18, CH-1015 Lausanne, Suisse \\ - e-mail : giovani.decesare@epfl.ch \\ 2. LCH, EPFL - e-mail : milad.daneshvari@epfl.ch \\ 3. LCH, EPFL - e-mail : matteo.federspiel@epfl.ch \\ 4. LCH, EPFL, maintenant chez Aecom, Montréal-e-mail : mathias.malquarti@a3.epfl.ch \\ 5. LCH, EPFL, maintenant au Laboratoire d'hydraulique environnementale (LHE),EPFL-e-mail : gael.epely-chauvin@epfl.ch \\ 6. LCH, EPFL - e-mail : anton.schleiss@epfl.ch
}

\begin{abstract}
RÉSUMÉ. - Le barrage de Koman, de $115 \mathrm{~m}$ de hauteur, est situé dans la partie nord de l'Albanie et fait partie des paliers hydroélectriques de la rivière Drin. L'aménagement dispose de deux évacuateurs de crue en tunnel avec des sauts de ski à leurs extrémités. Deux grandes fosses d'érosion se sont créées dans les alluvions avals. Le comportement hydraulique des deux évacuateurs et le processus d'affouillement ont été étudiés sur un modèle réduit et par modélisation numérique avec Flow-3D. L'écoulement dans les tunnels et sur les sauts de ski, ainsi que les trajectoires des jets et les zones d'impact ont pu être déterminés. L'évolution des fosses d'érosion et leurs étendues ont été étudiées. Les essais physiques ainsi que la modélisation numérique ont montré que, pour les deux évacuateurs, les sauts de ski ne peuvent guider les jets convenablement que jusqu'à $50 \%$ de leur capacité nominale. Au-delà, les sauts de ski ne peuvent plus dévier l'écoulement conformément aux prévisions théoriques, et la longueur du jet diminue, approchant la zone d'impact du pied du barrage. Les résultats de la modélisation physique et numérique pour l'état actuel ont pu être corroborés par les observations in situ. Le modèle physique et la simulation numérique permettent de reproduire fidèlement la création d'une fosse d'érosion dans les alluvions due à l'impact du jet plongeant.
\end{abstract}

Mots clefs : Fosse d'érosion, évacuateur de crue, saut de ski, modèle physique, modèle numérique

\section{Physical and numerical modeling of the spillways and plunge pools of Koman Dam in Albania}

\begin{abstract}
The $115 \mathrm{~m}$ high Koman Dam is located in the northern part of Albania and is part of the Drin River hydroelectric cascade. The dam is equipped with two tunnel spillways with flip buckets at their downstream limits. Two large scour holes were created in the downstream alluvium. The hydraulic behavior of the two spillways and the formation of the scour holes were studied on a physical scale model and by numerical simulation with Flow-3D. The flow in the tunnels and over the ski jumps, as well as the jet trajectories and the impact zones could be determined. The evolution of scour holes and their extent were studied. The physical model tests as well as the numerical simulations showed that, for the spillways, the flip buckets can deflect the jets suitably only up to $50 \%$ of the nominal capacity. Beyond that discharge, the flip buckets cannot guide the flow according to theory, and the jet length decreases, approaching the impact zone to the downstream dam toe. The results from the physical and numerical modeling for the current state could be validated against in situ observations. The physical model and the numerical simulation are able to reproduce accurately the plunge pool scour in an alluvial bed due to the plunging jet impact.
\end{abstract}

Key words : Plunge pool scour, spillway, ski jump, physical model, numerical model

\section{INTRODUCTION}

Le barrage de Koman est situé dans la partie nord de l'Albanie et est l'un des barrages existants des quatre paliers de la rivière Drin. C'est une digue à masque amont (et aval) en béton qui a été construit entre 1980 et 1988. La centrale hydroélectrique a une capacité installée de $600 \mathrm{MW}$. L'aménagement dispose de deux évacuateurs de crue principaux en tunnel avec des sauts de ski à leurs extrémités (Figure 1). L'évacuateur No. 3, situé sur la rive gauche, a une capacité de $1900 \mathrm{~m}^{3} / \mathrm{s}$. L'évacuateur No. 4 dont la capacité est de $1600 \mathrm{~m}^{3} / \mathrm{s}$ se situe en rive droite. Deux grandes fosses d'érosion se sont créées dans les alluvions à l'aval. Probablement lors des premières opérations de l'évacuateur No. 4, le pied aval du barrage a été érodé. La rive gauche qui sépare les fosses d'érosion du canal de fuite de la centrale hydroélectrique a également mal supporté la forte sollicitation hydraulique. En raison de ce problème, l'évacuateur No. 4 n'a presque plus été utilisé depuis.

Un des objectifs des essais sur modèle physique et numérique était d'identifier la source du problème d'instabilité au pied du barrage et de proposer des solutions. L'état actuel des deux évacuateurs No. 3 et No. 4 a été évalué et la géométrie des fosses d'érosion étudiée [1] [2] et [3]. 


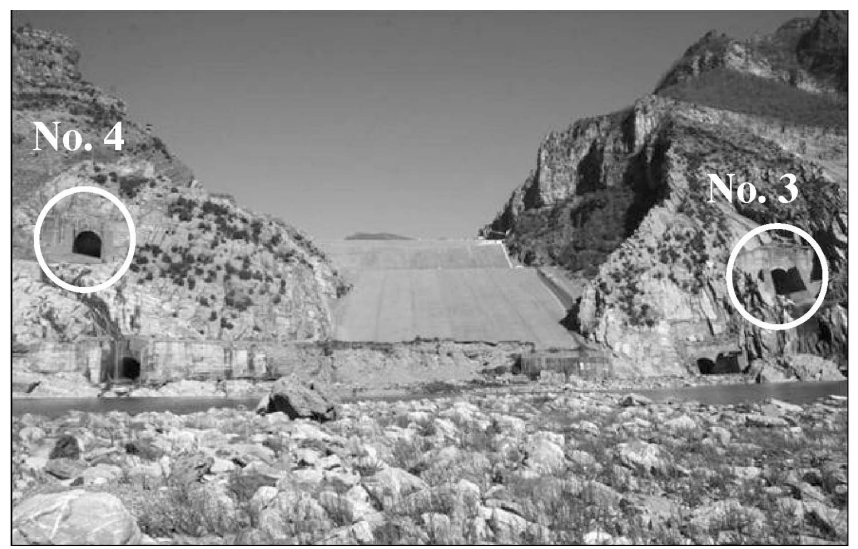

a)

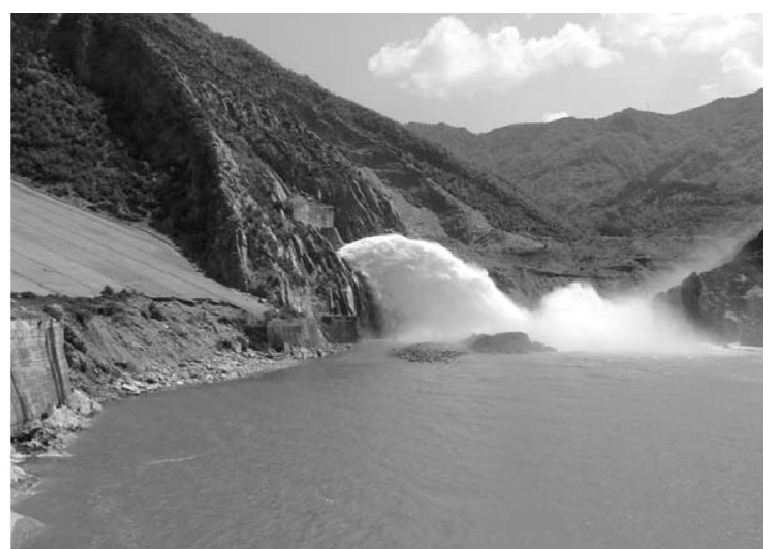

b)

Figure 1 : Vue de l'aval du barrage de Koman avec les deux sorties des évacuateurs de crue No. 4 et 3 et le pied aval érodé (a) ; photo de l'évacuateur No. 3 en rive gauche en fonction (b), photo G. De Cesare, 27.04.2010, Q $\approx 100 \mathrm{~m}^{3} / \mathrm{s}$.

\section{MODELLE PHYSIQUE}

Un modèle réduit hydraulique à fond mobile dans la partie de fosses d'érosion à l'échelle géométrique 1:65 a été construit au Laboratoire de Constructions Hydrauliques (LCH) de 1'Ecole Polytechnique Fédérale de Lausanne (EPFL) (Figure 2).

L'étude avait pour objectifs de :

- Vérifier l'écoulement dans les tunnels et aux sorties des évacuateurs de crues pour les différents scénarii d'ouverture des vannes et de débits,

- Mesurer et vérifier la trajectoire théorique du jet et de la zone d'impact dans le bassin amortisseur,

- Mesurer les niveaux d'eau dans le bassin amortisseur,

- Étudier qualitativement le mouvement de charriage et l'évolution potentielle des fosses d'érosion,

- Étudier la circulation de l'écoulement dans le bassin amortisseur, plus particulièrement au pied du barrage,

- Mesurer localement les vitesses dans le bassin amortisseur, plus particulièrement le long du pied du barrage et de la rive droite,
- Mesurer les pressions dynamiques agissant sur le pied du barrage.

En outre des modifications structurales des sauts de ski pour lesquelles la trajectoire du jet pourrait être améliorée ont été proposée, mais cette solution s'avérait irréaliste comme les ouvrages de sortie se trouvent directement à fleur du rocher.

La configuration du modèle du bassin aval ainsi qu'un photomontage sont présenté dans la Figure 2. Les profils des sections nécessaires à la construction ont été extraits de la topographie relevée sur place en 2008.

Dix différents scénarii de débits et de combinaisons des évacuateurs ont été définis pour les essais. Chaque évacuateur fonctionne individuellement à $25,50,75$ et $100 \%$ de sa capacité mais également ensemble, chacun à 50 et $100 \%$ de sa capacité nominale.

Des données de granulométrie proviennent de cinq relevés en ligne effectués in-situ dans les alluvions du bassin aval. Le matériel granulaire du prototype présente un $\mathrm{d}_{50}=11 \mathrm{~cm}$ et un $d_{90}=56 \mathrm{~cm}$, les plus grands blocs atteignent environ $1 \mathrm{~m}$ de diamètre. Le matériel sélectionné pour la modélisa-

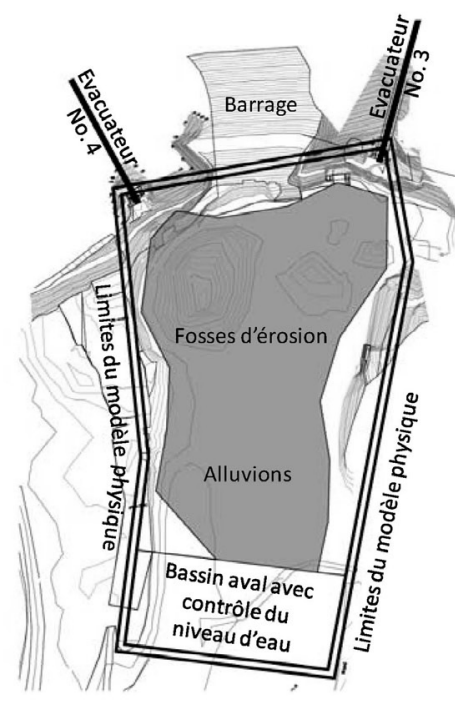

a)

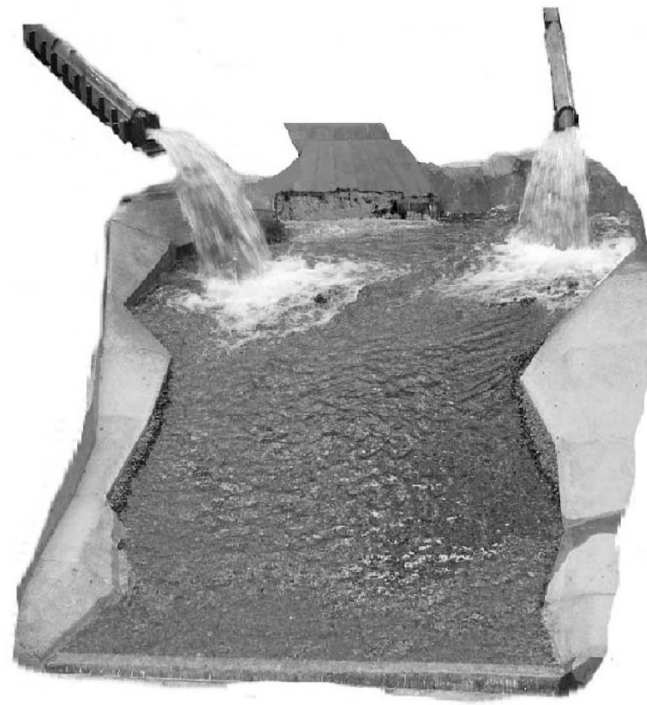

b)

Figure 2 : Limites et éléments du modèle physique (a) et photomontage des évacuateurs modèles en fonctionnement superposés à la photo du barrage réel (b). 
tion est composé de $50 \%$ de sable $8-11 \mathrm{~mm}$ et de $50 \%$ de gravier concassé $11-16 \mathrm{~mm}$ représentant les plus gros blocs. Le $\mathrm{d}_{50}$ est donc égal à $11 \mathrm{~mm}$. Ce mélange permet une bonne représentation de la réalité à l'échelle du modèle en ce qui concerne l'évolution et la profondeur de la fosse d'érosion selon Pagliara et al. (2008). Une fois les particules extraites de la fosse d'érosion, le monticule aval doit être évacué manuellement, car le transport solide ne se fait plus à l'aval. Une analyse du critère de Shields montre le bienfondé de la démarche.

Sur prototype le niveau d'eau dans le bassin amortisseur est directement lié au débit évacué, à un éventuel débit turbiné dans la centrale hydroélectrique en rive gauche, et au niveau du réservoir Vau-Dejës situé directement à l'aval. Un calcul de la courbe de remous depuis l'aval a été effectué sur la base de profils en travers disponibles et introduit à la section de contrôle à la sortie du bassin amortisseur. Le niveau d'eau est contrôlé par un déversoir situé dans le bassin à l'aval du modèle physique.(Figure 8a).

\section{MODÈLE NUMÉRIQUE}

Les simulations ont été effectuées avec le logiciel Flow-3D distribué par la société Flow Science [4]. Ce programme est autonome, gérant tant la partie solver, que le pre- que postprocessing, permettant la création de géométries, des conditions initiales et aux limites, la simulation numérique et la visualisation des résultats obtenus. Cependant, par souci de commodité, les éléments géométriques (évacuateurs, sauts de ski, topographie, barrage,...) ont été modélisés sous 3DSmax ou Autodesk Inventor puis importés dans Flow-3D.

L'étude numérique, effectuée à l'échelle du modèle physique, se composait de deux parties :

1. Implémentation des topographies obtenues avec le modèle physique comme fond fixe. Les conditions d'écoulement du modèle physique sont alors simulées. Les caractéristiques de l'écoulement, champs de vitesse et pression sont extraites.

2. Mise en place du modèle sédimentaire dans Flow-3D, tests de faisabilité à l'aide d'un cas d'étude de la littérature, puis simulation numérique avec fond mobile afin de retrouver les géométries obtenues dans le modèle physique, validées par l'observation in-situ.

Flow-3D résout numériquement les équations de continuité, d'énergie et de quantité de mouvement par une approche en volumes finis. Le domaine de simulation est intégré dans un maillage à cellules rectangulaires fixes. Toutes les variables sont calculées aux centres des cellules, à l'exception de la vitesse, qui est calculée sur les faces des cellules (staggered grid arrangement). Les obstacles et surfaces sont intégrés dans le maillage, en définissant sur chaque face du maillage une répartition entre une zone solide et une zone ouverte à l'écoulement (méthode dite Fractional Area-Volume Obstacle Representation - FAVOR). Cette technique permet une reproduction géométrique plus détaillée que la taille minimale des cellules du maillage.

Le choix de la dimension des mailles est toutefois important. Diminuer la taille des mailles permet d'augmenter la précision (de l'écoulement et des géométries) mais pénalise également le temps de calcul. Afin de minimiser le nombre de cellules tout en gardant une bonne précision, un maillage composé de plusieurs blocs a été utilisé (Figure 3b).

La continuité entre deux blocs adjacents est assurée directement par Flow-3D, pour autant que l'on ne dépasse pas 5 à $10 \%$ de différence entre la taille des cellules qui composent deux blocs contigus. Un rapport hauteur/largeur/profondeur des cellules proche de l'unité a été choisi pour l'ensemble des blocs, garantissant une stabilité numérique. Une prévisualisation interactive des solides et du fluide à l'état initial est possible dans Flow-3D grâce au module FAVOR. Il permet de bien optimiser le maillage avant de lancer la simulation (Figure 3b).

Les maillages utilisés varient en fonction des scénarii : de 900'000 cellules pour les simulations mettant en jeu un seul évacuateur de crue avec forme des fosses d'érosion prédéfinie et jusqu'à 2'000'000 cellules pour l'ensemble barrage - évacuateurs de crue - bassin amortisseur aval avec fond érodable (Figure 4a).

Les caractéristiques physiques du problème ont été définies en fonction des paramètres à analyser, les deux modèles de base étant le modèle gravitaire et le modèle de viscosité/ turbulence. Pour la turbulence, le modèle k- $\varepsilon$ a été implémenté. Ce modèle permet d'obtenir des approximations correctes des écoulements à fort Reynolds, ce qui est le cas ici. Pour l'opérateur d'advection (l'advection correspond au transport d'une quantité scalaire conservée par un champ vectoriel), une approximation du $3^{\text {ème }}$ ordre a été implémentée afin de permettre l'étude d'écoulements tourbillonnaires. Pour la simulation cohérente d'un jet d'eau, le modèle tient également compte de la tension superficielle de l'eau.

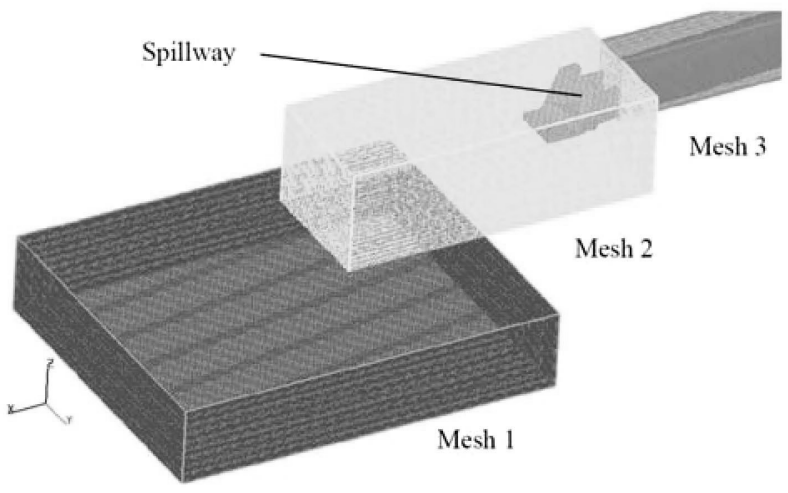

a)

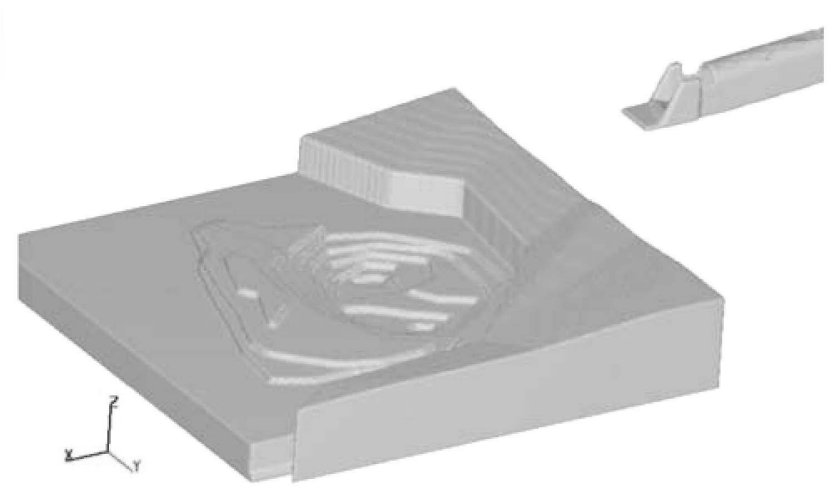

b)

Figure 3 : Modèle numérique Flow-3D en 3 blocs pour le modèle partiel de l'évacuateur No. 4 avec la galerie, la zone du jet et la fosse d'érosion à l'aval (a) et résultant du rendu géométrique final par le module FAVOR (b). 


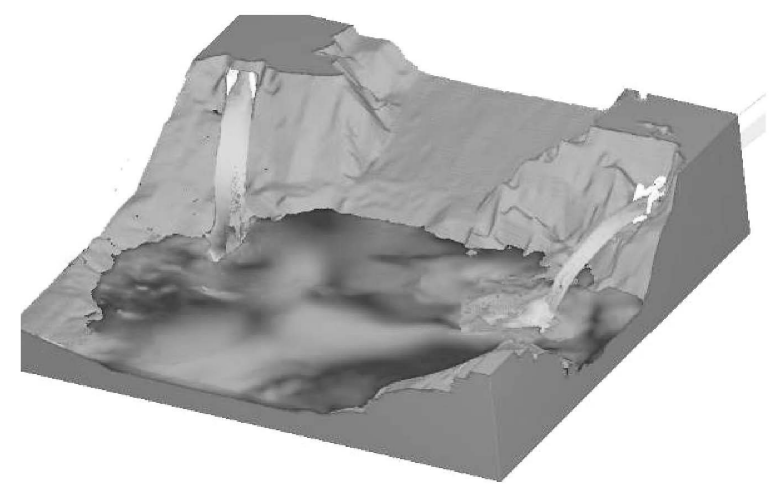

a)

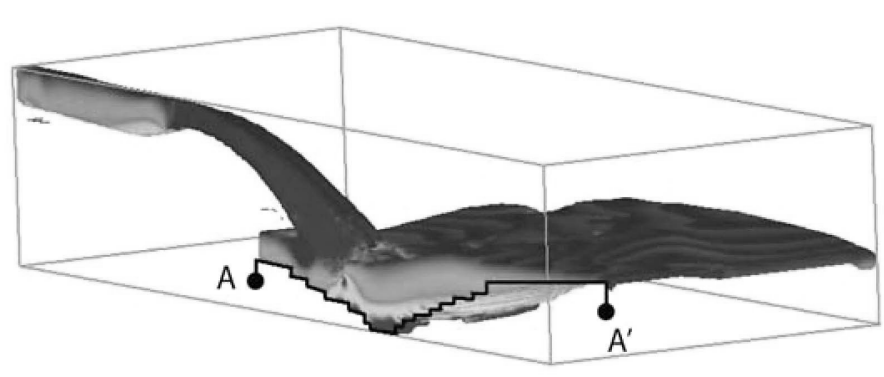

b)

Figure 4 : Modèle numérique Flow-3D de l'ensemble barrage - évacuateurs de crue - bassin amortisseur aval (a) et modèle partiel pour l'étude de l'évacuateur No. 3 montrant l'écoulement dans la galerie, le jet et une coupe à travers la fosse d'érosion aval (b).

Les conditions aux limites à l'amont (dans les évacuateurs) sont les vitesses et les hauteurs d'eau imposées à l'aval des vannes en fonction du débit et de l'ouverture des vannes. La condition aval est définie comme une section à niveau d'eau constant, avec une distribution hydrostatique des pressions du fluide sortant.

Pour le modèle numérique à fond mobile, la même géométrie et les mêmes conditions amont et aval sont définies. Un lit formé de sédiments a été mis en place sur un fond fixe plus profond. Les caractéristiques des sédiments sont identiques à celles du modèle physique, soit une masse spécifique $\rho=2650 \mathrm{~kg} / \mathrm{m}^{3}$ et un angle de frottement $\phi=35^{\circ}$. La granulométrie non-uniforme, mais peu étendue, a été prise en compte avec un diamètre moyen $\mathrm{d}_{\mathrm{m}}=11 \mathrm{~mm}$. Les deux évacuateurs ont chacun fonctionné à $100 \%$ de leur capacité nominale.

\section{RÉSULTATS}

\section{IV.1. Trajectoire des jets}

Pour tous les scénarii, la forme et les trajectoires de la nappe inférieure et supérieure ont été mesurées sur le modèle physique. Le modèle numérique fournissait, pour comparaison, le résultat pour $100 \%$ de capacité uniquement.

A titre d'exemple, la forme du jet de l'évacuateur No. 3 à $100 \%$ de sa capacité est présenté Figure 5. Les résultats numériques sont globalement en accord avec le modèle phy- siqueb). L'étalement du jet augmente avec les débits. Il reste à noter une très forte influence de la taille du maillage sur la précision de la simulation des jets. Un contrôle des flux a permis de vérifier que le jet ne "perdait» pas de l'eau, la continuité étant satisfaite dans le modèle numérique. C'est essentiellement au niveau de l'affichage des résultats lors du postprocessing que des «trous » apparaissaient dans les jets.

Il y a deux critères géométriques principaux pour la conception d'une auge d'un saut de ski [5]: le rayon de l'auge $\mathrm{R}$ et son angle d'ouverture $\beta$ (Figure 6). Le rayon $\mathrm{R}$ de l'auge devrait être d'au moins quatre fois la profondeur d'eau à l'approche, $\mathrm{h}_{0}$. En outre, l'auge devrait être capable de guider le jet convenablement avec un débit spécifique de l'ordre de $110 \mathrm{~m}^{3} / \mathrm{s} / \mathrm{m}$. Le deuxième critère est l'angle d'ouverture de l'auge, $\beta$, qui varie normalement entre $25^{\circ}$ et $35^{\circ}$. L'angle de détachement de l'auge $\alpha$ est en règle générale égal à l'angle d'ouverture, sauf si la profondeur d'eau dépasse le critère du rapport $R / h_{0}>4$.

En fonction des caractéristiques hydrauliques de l'écoulement et de la géométrie du saut de ski, la trajectoire théorique peut être calculée [6] [7]. Le résultat de ce calcul est montré à la Figure 7 et est comparé aux mesures effectués sur le modèle physique. Il en sort clairement, que la trajectoire observée sur modèle physique donne un jet nettement plus court que prédit théoriquement, cela pour des débits dépassant $50 \%$ de la capacité des évacuateurs de crue. Dans le cas de l'évacuateur No. 4 à $100 \%$ de sa capacité, la distance depuis le point de départ au centre de l'impact du jet est réduite jusqu'à $12 \mathrm{~m}$ avec l'angle de départ réduit, ce qui

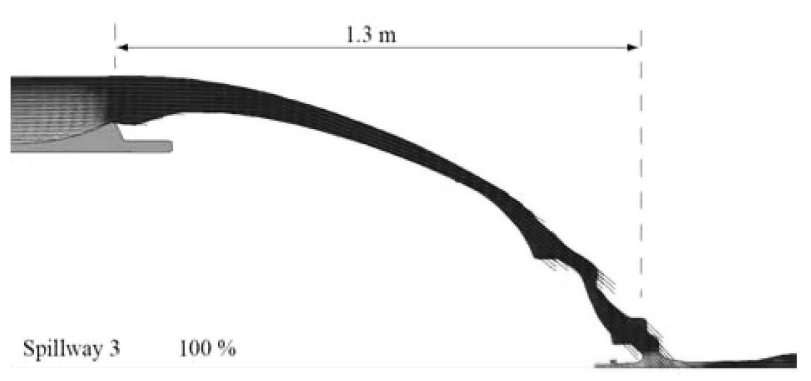

a)

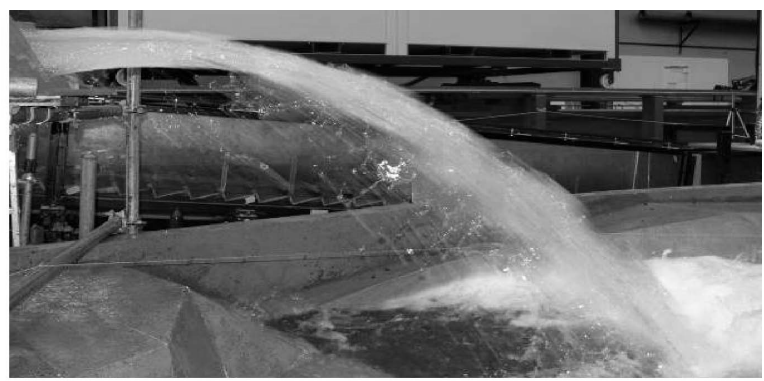

b)

Figure 5 : Trajectoire du jet pour l'évacuateur No. 3 à $100 \%$ de sa capacité, $1900 \mathrm{~m}^{3} / \mathrm{s}$. La compression de la nappe supérieure du jet sur le saut de ski est bien visible, réduisant sa longueur et approchant ainsi la zone d'impact du jet vers le barrage; résultats de la modélisation numérique (a) et physique (b). 


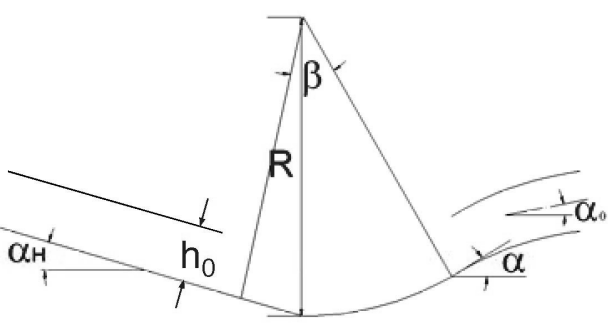

$\alpha:$ angle de détachement de l'auge

$\alpha_{0}$ : angle du jet

$\alpha_{H}$ : pente du fond du coursier amont

$\beta$ : angle d'ouverture de l'auge

$\mathrm{h}_{0}$ : profondeur d'eau dans le coursier amont

$\mathrm{R}$ : rayon de l'auge

Figure 6 : Définition et schéma des caractéristiques géométriques d'un saut de ski

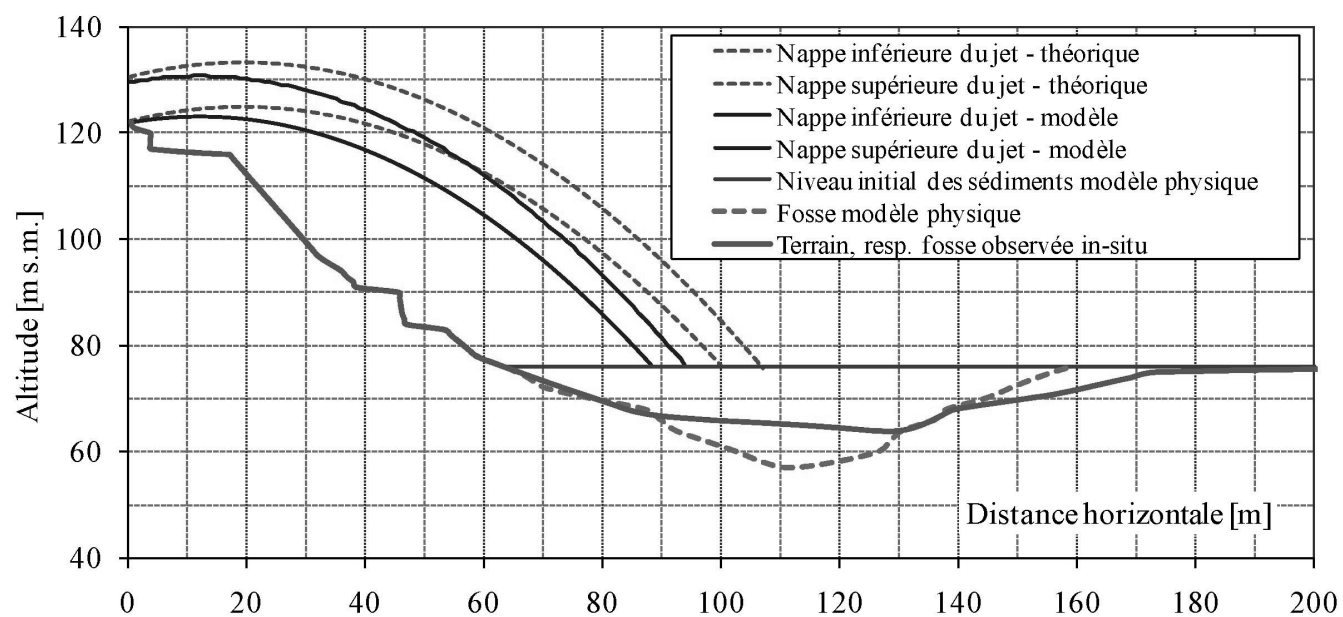

Figure 7 : Trajectoires observées sur modèle physique et théoriques pour l'évacuateur No. 4 à $100 \%$ de sa capacité, $1600 \mathrm{~m}^{3} / \mathrm{s}$. Dans ce cas, la distance au centre de l'impact du jet est réduite jusqu'à $12 \mathrm{~m}$, ce qui est confirmé par les observations in-situ.

est confirmé par les observations in-situ, notamment par la position et la forme de la fosse d'érosion.

\section{IV.2. Zone d'impact des jets}

Dans cette partie de l'étude la zone d'impact du jet et sa forme ont été mesurées. Pour réaliser les mesures, une grille en fils synthétiques a été placée légèrement en dessus du niveau de l'eau (Figure 8).

Le maillage de la grille est de $10 \times 10 \mathrm{~cm}^{2}$. Cette grille a permis de délimiter schématiquement la forme du jet dans sa zone d'impact. Cette mesure a été effectuée pour les quatre débits de chaque évacuateur. Des mesures ponctuelles de la pression à l'intérieur du jet à l'impact ont également été effectuées.

La Figure 9 illustre schématiquement la zone d'impact. La zone d'émission du jet (la fin du saut de ski) y est également indiquée pour une meilleure compréhension. La zone d'impact du jet correspond à une forme de volute. Le noyau du jet (core jet) n'est plus clairement identifiable. La forme observée du jet évolue vers un aplatissement avec l'augmentation du débit (voir également Figure 5).

Cette trajectoire est due à la forme du saut de ski avec sa lame coupée en biais ainsi qu'à la profondeur d'eau pour chaque débit. Il a été observé que plus la profondeur d'eau augmente (donc plus le débit augmente) moins la nappe

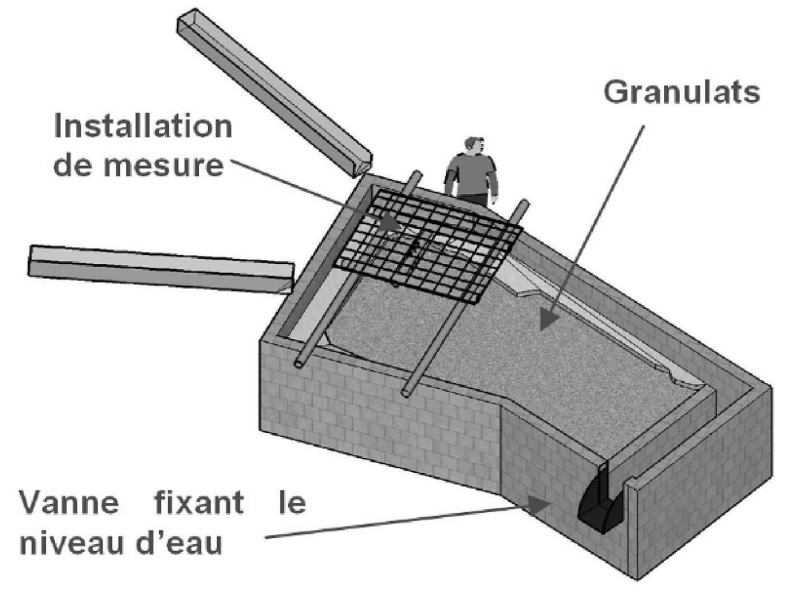

a)

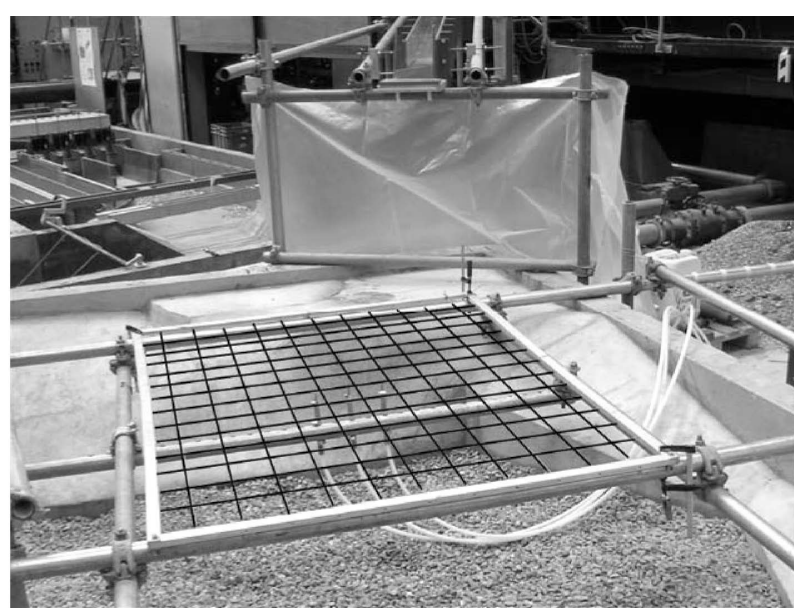

b)

Figure 8 : Dispositif permettant de délimiter la zone d'impact du jet et d'y mesurer les pressions 


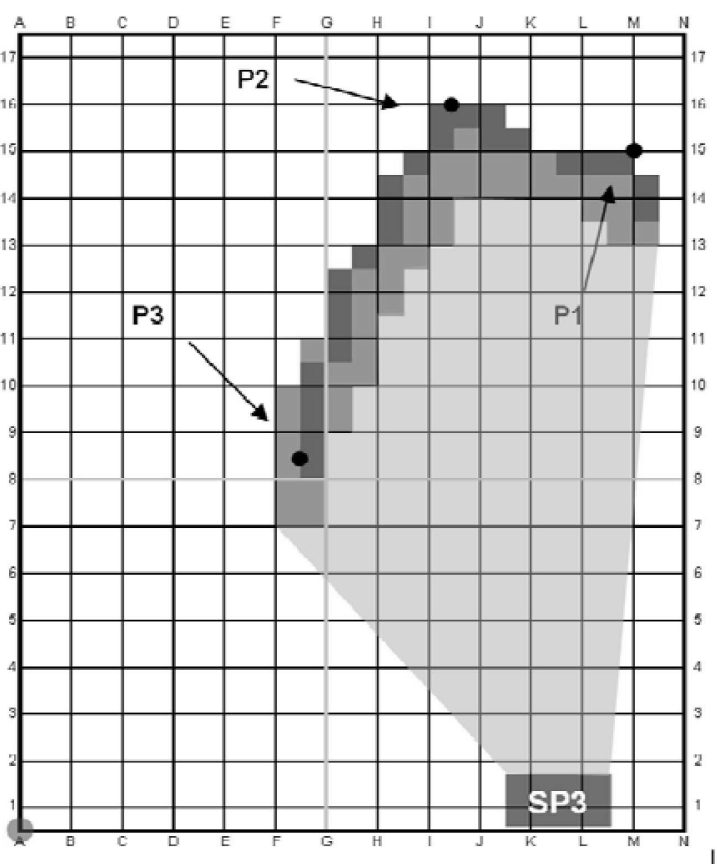

a)

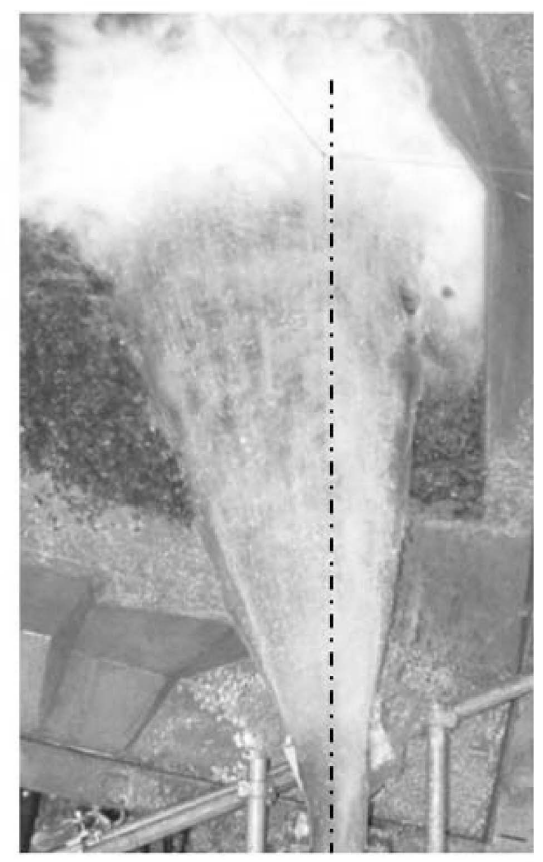

b)

Figure 9: Schéma de la forme typique sur modèle physique de la zone d'impact du jet pour l'évacuateur No. 3 à $50 \%$ de sa capacité, $950 \mathrm{~m}^{3} / \mathrm{s}$ (a). La fin du saut de ski SP3 comme origine du jet est également indiquée. Les cercles noirs représentent l'emplacement des mesures de pression sous le jet dans la zone d'impact. Un carré couvre $10 \times 10 \mathrm{~cm}^{2}$. Photo du jet vu pardessus l'évacuateur No. 3 montrant la dispersion du jet décollant du saut de ski et délimitant la forme caractéristique de la zone d'impact (b).

supérieure du jet subit l'effet déviateur du saut de ski, raison pour laquelle le jet perd de sa longueur et s'aplatit pour prendre une forme plus proche d'un jet rectangulaire. On peut dès lors remarquer que la forme du jet évolue considérablement par rapport à la forme de départ durant sa chute.

\section{IV.3. Fosses d'érosion}

Durant les essais menés dans le cadre de ce projet, les fosses d'érosion (forme, profondeur d'affouillement et hauteur des dépôts aval) ont été relevées pour les différents débits et combinaisons des évacuateurs. Les résultats obtenus avec le modèle sédimentaire de Flow-3D sont présentés et comparés aux résultats de la modélisation physique. Ces résultats ont été validés par les relevés in-situ, concernant essentiellement la position, la forme et la profondeur de la fosse d'érosion de l'évacuateur No. 4 car celle-ci se trouve intégralement dans les alluvions meubles.

Avant de réaliser les simulations d'érosion dans le modèle physique, des tests concernant la capacité de Flow-3D à pouvoir simuler l'érosion et le transport solide résultant d'un jet plongeant ont été effectués. Ainsi les expériences de Pagliara et al. (2008) [8], portant sur l'évolution temporelle de l'affouillement d'un lit sédimentaire dû à un jet plongeant ont été modélisées, simulées et les résultats validés. Ensuite, le modèle sédimentaire de Flow-3D a été utilisé pour simuler l'érosion au pied des deux évacuateurs de crue fonctionnant ensemble. Les résultats des géométries obtenues par simulation numérique et sur le modèle physique sont donnés à la Figure 10. La localisation ainsi que le développement spatial des zones d'affouillement et des dépôts aval sont très proches.
Le Tableau 1 synthétise les profondeurs d'érosion et les hauteurs des dépôts à l'aval des fosses. Les profondeurs d'érosion obtenues sont en accord avec les essais physiques.

Pour des raisons de temps de calcul élevé (le temps de simulation est de l'ordre de $15 \mathrm{~min}$ correspondant à environ 15 jours de calculs CPU pour chaque test), les essais numériques avec modèle de transport solide ont été relativement courts, la profondeur ultime d'érosion n'étant probablement pas encore atteinte. La durée des essais physiques était de 45 minutes. La hauteur du dépôt maximal aval correspond au monticule pour les essais où ce dernier n'a pas été enlevé. Une fois les particules extraites de la fosse, elles ne peuvent pas être transportées plus loin, d'où le monticule relativement élevé dans le modèle physique, formé essentiellement par les particules les plus grossières. Dans le modèle numérique, un seul diamètre représentatif $\mathrm{d}_{\mathrm{m}}$ a été utilisé, inférieur au $d_{\max }$ du modèle physique et donc plus facilement mobilisable, d'où une hauteur du dépôt maximal aval plus faible dans le modèle numérique.

\section{CONCLUSIONS}

Les deux évacuateurs de crue ainsi que les différentes fosses d'érosion du barrage de Koman en Albanie ont été étudiés sur un modèle hydraulique à l'échelle $1: 65$ et par modélisation numérique avec Flow-3D. L'étude portait notamment sur l'écoulement dans les auges des sauts de ski, les trajectoires des jets ainsi que sur les zones d'impact et les fosses d'affouillement.

Les résultats de l'étude mixte, numérique et physique, ont montré que, pour les deux évacuateurs, les sauts de ski ne peuvent plus dévier les jets convenablement au-delà de $50 \%$ de leur capacité. La déflection selon les prévisions 


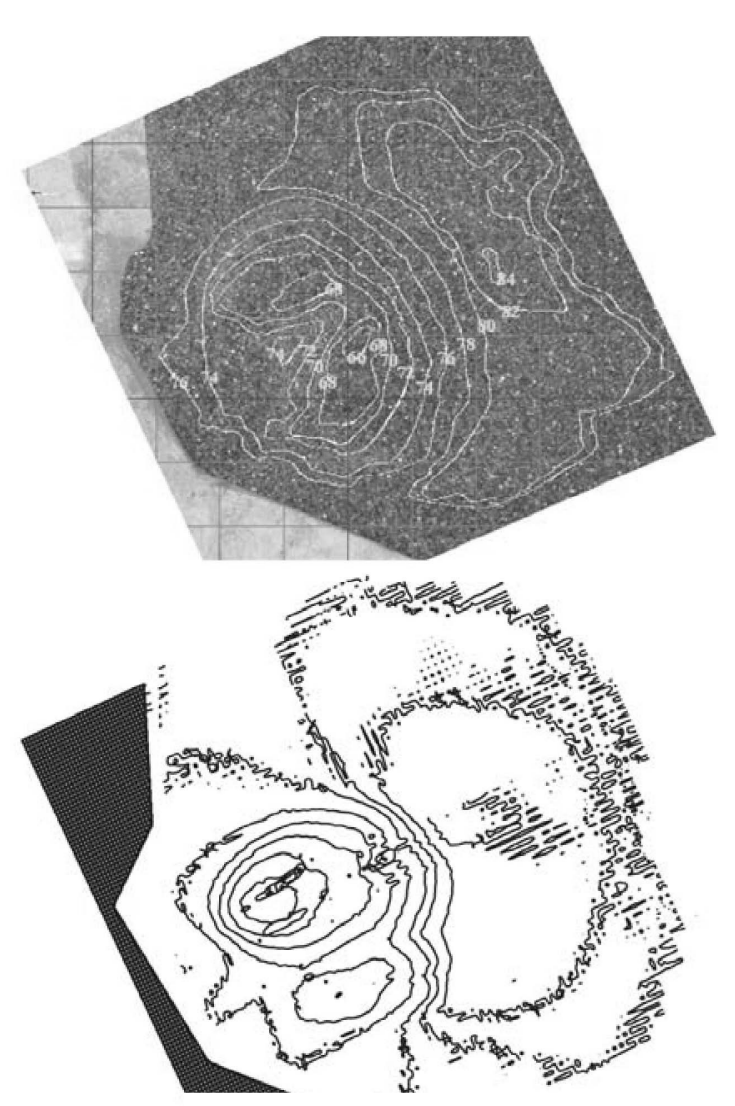

évacuateurNo. 4

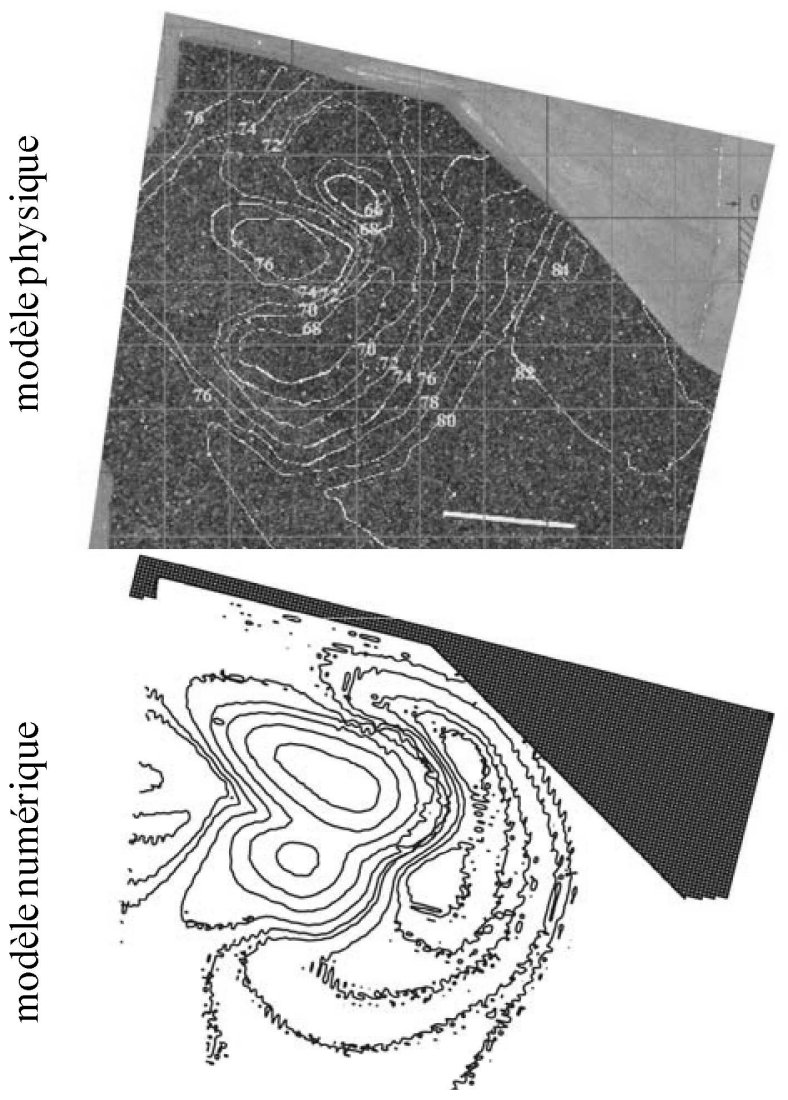

évacuateur No. 3

Figure 10 : Comparaison des géométries des fosses d'érosion à l'aval des évacuateurs de crue entre le modèle numérique et le modèle physique, fonctionnant à $100 \%$ de leur capacité. Les fosses ainsi que les dépôts aval ont été simulés correctement en termes de position, profondeur, étendue et volume.

Tableau 1 : Comparaison entre les géométries issues du modèle numérique et du modèle physique à l'échelle du modèle pour les évacuateurs No. 4 et 3 fonctionnant à $100 \%$.

\begin{tabular}{|l|c|c|c|c|}
\hline & \multicolumn{2}{|c|}{ Profondeur d'érosion [m] } & \multicolumn{2}{c|}{ Hauteur du dépôt aval [m] } \\
\hline Résultats de la modélisation & numérique & physique & numérique & physique \\
\hline Fosse d'érosion à l'aval de l'évacuateur No. 4 & 0.135 & 0.108 & 0.092 & 0.123 \\
\hline Fosse d'érosion à l'aval de l'évacuateur No. 3 & 0.138 & 0.154 & 0.013 & 0.123 \\
\hline
\end{tabular}

théoriques ne se fait plus, la longueur du jet diminue, la zone d'impact et la fosse d'érosion s'approchent du pied du barrage. Les résultats de la modélisation physique et numérique ont été validés par les observations in situ. Le modèle physique et la simulation numérique permettent de reproduire fidèlement la création d'une fosse d'érosion dans des alluvions résultant de l'impact d'un jet plongeant.

Malgré de nombreuses simplifications du modèle numérique (en particulier l'effet d'aération et la limite due au maillage) et malgré les effets d'échelle du modèle physique, cette approche hybride numérique et physique permet de mieux comprendre les phénomènes en jeu. De plus, elle permet de prédire l'effet d'une éventuelle mesure à prendre au niveau du prototype comme la modification de l'auge du saut de ski afin de garantir à long terme la stabilité du barrage et le bon fonctionnement des évacuateurs de crue.

Lors des crues de fin 2010 en Albanie, le pied du Barrage de Koman a subi à nouveau de fortes érosions. Suite à ces événements, des mesures de stabilisation du pied de digue ont été dimensionnées et proposées au propriétaire. Une simulation sur modèle numérique sans tenir compte de fond mobile et sans sédiments (clear water) a été effectuée à l'échelle prototype pour déterminer la sollicitation hydraulique au pied du barrage à l'état actuel et futur avec la profondeur ultime des fosses d'érosion [9].

Comme dans toute modélisation, qu'elle soit numérique ou physique, et tout particulièrement dans le domaine du transport solide, les résultats sont à considérer comme des valeurs qualitatives, la quantification exacte étant plus difficile à atteindre. Malgré les efforts de l'ingénieur expérimentateur et l'application stricte des lois de similitudes hydrauliques (et de celles du transport solide), la transposition des résultats et des conclusions reportées au prototype nécessitent beaucoup d'expérience et de savoir-faire. La validation par des observations sur modèle physique ou prototype est indispensable pour obtenir des résultats qualitatifs fiables par la modélisation numérique. Un sens critique, du talent 
d'interprétation et une mise en question de ce qui est fait demeurent indispensables. Néanmoins, les outils et méthodes à disposition de l'ingénieur-chercheur aujourd'hui permettent de faire de la modélisation couplée physique et numérique un outil éprouvé pour un transfert avec succès des résultats vers l'application pratique.

\section{REMERCIEMENTS}

L'étude a été réalisée pour le propriétaire et les autorités locales, Koman HPP et l'Albanian Power Corporation KESH à Tirana, ainsi que pour leur consultant Pöyry Energy Ltd. à Zürich. Le projet est financé par la Direction pour le développement et la coopération (DDC) du Secrétariat d'Etat à l'économie Suisse (SECO), la Banque Mondiale et la KfW Förderbank dans le cadre du projet de réhabilitation du barrage de Koman.

\section{RÉFÉRENCES}

[1] Epfl-Lch (2009) - Koman Dam Rehabilitation Project. Hydraulic model tests for spillways and plunge pool. Report on physical model, Rapport d'étude du LCH, non publié. 2
[2] Epely Chauvin G. (2009) - Dimensionnement de bassins de dissipation d'énergie non-revêtus pour des grands barrages en béton à l'aide de modélisation 3D. Travail de Master en Génie Civil, EPFL-ENAC-LCH

[3] Malquarti M. (2009) - Étude de pressions générées par des jets à hautes vitesses, mesures en laboratoire et étude de cas. Travail de Master en Génie Civil, EPFL-ENAC-LCH

[4] Flow Science (2008) - Flow3D User's Manual - Theory, Flow Science Flow $3 D$ v9.3

[5] Asce (1995) - Hydraulic design of spillways. Technical Engineering and Design Guides as adapted from the U.S. Army Corps of Engineers, American Society of Civil Engineers. 12

[6] Viatcheslav O. (1974) - Die Bestimmung des Strahlsteigwinkels beim Abfluss über einen Sprungschanzenüberfall. Wasserwirtschaft-Wassertechnik. 24(9)

[7] KaWAKami K. A (1973) - Study on the computation of horizontal distance of jet issued from ski jump spillways. Trans. of the Japanese Society of Civil Engineers

[8] Pagliara S., Hager W. H., And Unger J. (2008) - Temporal Evolution of Plunge Pool Scour. Journal of Hydraulic Engineering. 134(11) 1630-1638

[9] Epfl-Lch (2010) - Koman Dam Rehabilitation Project. Numerical 3D simulation for spillways and plunge pool hydraulics. Rapport d'étude du LCH, non publié. 34 